\title{
INCREASING THE THROUGHPUT OF LTE USING DEVICE-TO-DEVICE COMMUNICATION BY REDUCING INTRA-SYSTEM INTERFERENCE FLOW
}

\author{
Adel F. Rakhimov, \\ Dept. of Radioelectronic and Telecommunication Systems, \\ Kazan National Research Technical University named after A. N. Tupolev-KAI, City of Kazan, Russia; \\ German-Russian Institute of Advanced Technologies (GRIAT), Rakhimov2897@yandex.ru \\ Anna A. Bukharina, \\ Dept. of Radioelectronic and Telecommunication Systems, \\ Kazan National Research Technical University named after A. N. Tupolev-KAI, City of Kazan, Russia; \\ German-Russian Institute of Advanced Technologies (GRIAT), AABukharina@kai.ru
}

DOI: $10.36724 / 2664-066 X-2020-6-4-3 \mid-35$

\begin{abstract}
This article focuses on the using collective dynamic route method for D2D LTE networks. It provides algorithms for the analysis stage, taking into account the availability of D2D users and the routing stage, as well as their implementation. The dependencies of the D2D communication routes quantity fraction for various cases of user location are shown.
\end{abstract}

KEYWORDS: collective dynamic routing, one-dimensional routes, multidimensional routes, Bounded Recurrent Algorithm.

\section{INTRODUCTION}

To increase the throughput of broadband radio access networks by reducing intra-system interference flow in [1], a collective dynamic routing method was developed. It includes two stages:

1) The analysis stage includes following steps:

- Constructing set of routes;

- Calculating signal power for each route;

- Calculating signal-to-interference ratio (SINR) for each route for each channel;

- Calculating data rate with a given error probability for each route and each channel.

2) Routing stage that includes defining the optimal set of routes that provides data transmission with minimal delivery time.

As described in [3], this method allows increase the IEEE 802.11 networks throughput.

However, the researches did not consider the applying possibility this method for LTE networks using D2D communication. Features of D2D communication require a change in the algorithm for searching acceptable route set.

So, this article is devoted to the development of the algorithm for searching acceptable route set for LTE networks with D2D communication and its effectiveness analysis. 


\section{PROBLEM STATEMENT}

Consider LTE network example consisting of one base station (BS) and three users (Figure 1).

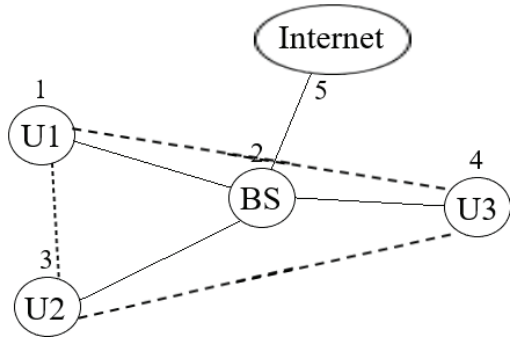

Fig. 1. The LTE network example

The task of this network is to deliver data between users $\mathrm{U} 1$ - U3, as well as between them and the Internet, i.e. deliver data in volumes specified by the matrix I accumulated in the interval $T^{I}$ using acceptable route $\operatorname{set}\{\mathbf{w}\}$.

The collective dynamic routing method allows to find the optimal route set $\vec{N}^{\text {opt }}$, as the number of using frames for each of the route in the set $\{\mathbf{w}\}$, which deliveries data with the volumes $\mathbf{I}$ for the minimum time according to:

$$
\left\{\begin{array}{l}
\vec{N}^{o p t}=\underset{\vec{N}}{\arg \min _{\vec{N}}}\left(\sum_{g=1}^{G} N_{g}\right) \\
\sum_{g=1}^{G} N_{g} \cdot \widetilde{I}_{g n^{R} n^{T}} \geq I_{n^{R} n^{T}}, \quad n^{R}=\overline{1, N}, n^{T}=\overline{1, N}, \\
N_{g} \geq 0, \quad g=\overline{1, G} \\
N_{g} \in Z,
\end{array}\right.
$$

where $\vec{N}=\left(N_{1}, \ldots, N_{g}, \ldots, N_{G}\right)$ is the used route set; $G$ is the total routes quantity; $n^{R}$ is the receiving node in route $w_{g} ; n^{T}$ is the transmitting node in route $w_{g} ; N$ is the total transmitting or receiving nodes quantity.

Thus, depending on the volumes matrix I, the optimal routing set will be different.

\section{ANALYSIS STAGE}

According to collective dynamic routing method, the acceptable route set consists of certain amounts of endto-end routes that can be divided into one-dimensional and multidimensional routes.

One-dimensional route is set of serially connected communication channels in order to connect desirable users. All one-dimensional routes can be divided into three groups: routes that allow users to access the Internet and routes connecting different users which are into routes connecting users through the BS and directly (D2D). For the considering LTE network example the first group contains routes: (1-2-5), (3-2-5) and (4-2-5), the second group - routes: (1-2-3), (1-2-4), (3-2-1), (3-2$4),(4-2-1),(4-2-3)$ and third group - routes: (1-3), (1-4), $(3-1),(3-4),(4-1),(4-3)$.
Multidimensional routes are obtained by unification of one-dimensional routes that use different communication channels. Two dimensional routes includes two one-dimensional routes, e.g. \{(1-2-3), (3$4)\}$. Three dimensional routes includes three onedimensional routes, e.g. $\{(1-2-3),(3-2-4),(4-3)\}$.

The implementation was done in Matlab. After routes construction and removing redundant routes, such as (1-2-5) and (1-5), for the example shown in figure 1215 routes were obtained.

The signal powers $P_{g n^{R} n^{T}}$ were calculated depends on the propagation loss described by ITU-R 1411 [4] for each route in set $\{\mathbf{w}\}$ and each user pairs. The transmitter powers of BS and user equipment were determined based on the standard for LTE and LTE-Advanced dated October 29, 2018 [5].

According to [6] SINR $\rho_{\operatorname{gin}^{R} n^{T}}^{R^{S}}$ is calculated such way:

$$
\rho_{g i n^{R} n^{T}}^{R^{S}}=\frac{P_{g n^{R} n^{T}}}{P_{n} \cdot\left(1+K_{g i n^{R}}^{N R^{S}}\right)+\sum_{n^{T}=1}^{N^{T}} P_{g n^{R} n^{n^{T}}} \cdot \sum_{i^{\prime}=1}^{I^{u}} K_{g i i^{\prime} n^{R} n^{t^{T}}}^{P R^{S}}},
$$

where $\rho_{g n^{R} n^{T}}$ is a SNR at $n^{R}$-th user receiver from $n^{T}$-th user transmitter; $K_{g i n^{R}}^{N R^{S}}$ and $K_{g i i^{\prime} n^{R} n^{\prime T}}^{P{ }^{S}}$ are noise and intrasystem an interference influence coefficients with calculated with program OFDM Receiver [7].

In order to reduce interference, we should think about resource blocks distribution between routes. The algorithm for resource blocks distribution in onedimensional routes is shown in figure 2.

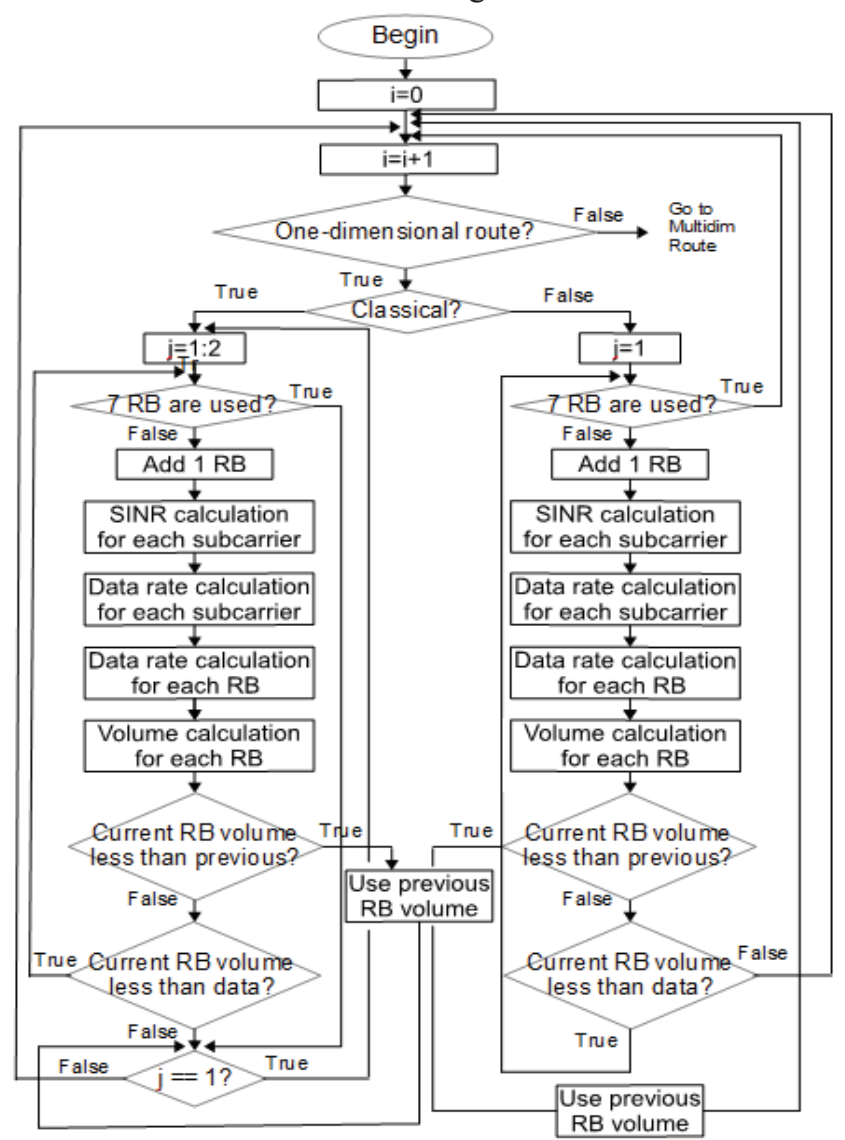

Fig. 2. Algorithm of one-dimensional routes analysis stage 
As can be seen, the distribution is done based on the total number of resource blocks and the comparison of current and previous values of volume that is delivered by the total used resources per route. If current value of volume is less than previous, it denotes that interference level when we use current distribution is much higher in comparison with interference level when we use previous distribution and, as a result, previous distribution gives more efficient results in data rate terms.

The same approach used for two and three multidimensional routes but algorithm will be more complicated because in this case we should remember about finite number of resource blocks, namely 7 , and the variety of the variants of data delivery. In two dimensional routes there are 3 variants, e.g. only classical approach: $\{(1-2-3),(3-2-4)\}$; classical approach and D2D: $\{(1-3),(3-2-4)\}$ or $\{(1-2-3),(3-4)\}$ and D2D only $\{(1-3),(3-4)\}$. In three dimensional routes case we have such variants: only classical approach; two routes with classical approach and one route with $\mathrm{D} 2 \mathrm{D}$; one route with classical approach and two routes with D2D; D2D only.

SINR $\rho_{\operatorname{gin}^{R} n^{T}}^{R^{S}}$ for each subcarrier is different. Therefore data rate for each subcarrier will be different too. In this case the channel's data rate from $n^{T}$-th to $n^{R}$-th user is calculated by following expression:

$$
V_{g n^{R} n^{T}}\left(R^{s}\right) \leq 2 \cdot V^{t e c} \cdot \sum_{i=1}^{I^{u}} \log _{2}\left(\frac{\sqrt{\rho_{g i n^{R} n^{T}}^{R^{S}}}}{2 \cdot Q^{-1}\left(P_{\max }^{E r} / 2\right)}+1\right)
$$

where $V^{\text {tec }}$ is an amount of transmitted OFDM-symbols per second; $P_{\max }^{E r}$ is transmitting symbol error probability; $I^{u}$ is an amount of subcarriers that are used for data delivery.

The volume $\widetilde{I}_{g n^{R} n^{T}}$ that can be delivered by one resource block from $n^{T}$-th to $n^{R}$-th user along the route $w_{g}$ can be found as production of the channel's data rate by the resource block delivery time $T^{F}$ :

$$
\widetilde{I}_{g n^{R} n^{T}}=V_{g n^{R} n^{T}} \cdot T^{F} .
$$

The choice of routes used for the data delivery is carried out at the routing stage based on data volumes $I_{n^{R} n^{T}}$ at matrix $\mathbf{I}$, and volumes $\widetilde{I}_{g n^{R} n^{T}}$ delivered by one resource block

\section{ROUTING STAGE}

The aim of this stage is finding optimal route set $\vec{N}^{\text {opt }}$. In the general, $\vec{N}^{\text {opt }}$ can be obtained by solving system (1), which is the task of integer linear programming (ILP). However, due to the high complexity of the exact solution ILP task, recurrent algorithm can be used.
Based on the algorithms comparison that can be used in the collective dynamic routing method described in [8], given the complexity of the calculations and the obtained data delivery time, the Bound Recurrent Algorithm (BRA) was been choose. This recursive algorithm at each step determines the number of the best route, which can be determined as follows:

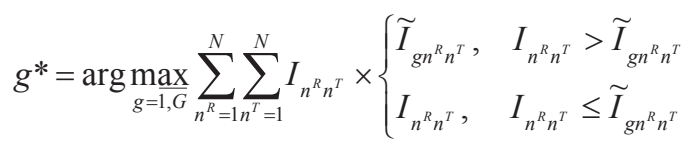

where $w_{g^{*}}$ is the best route with number $g^{*} ; N$ is the user quantity; $I_{n^{R} n^{T}}$ is amount of data, which needed to transmitted by user $n^{T}$ to user $n^{R} ; \widetilde{I}_{g n^{R} n^{T}}$ is amount of data transmitted by user $n^{T}$ to user $n^{R}$ at the resource block delivery time $T^{F}$ using route $w_{g}$.

So, $w_{g^{*}}$ computes in $T^{F}=0.5 \mathrm{mc}$ every time until all data is transmitted. The sequence of $w_{g^{*}}$ give us the vector of optimal route set $\vec{N}^{\text {opt }}$.

\section{RESULTS}

Four cases were considered eo evaluate the effectiveness of using D2D communications.

The first case considers all users are placed close to $\mathrm{BS}$ and at equal distances from each other. It is shown in Figure 3.

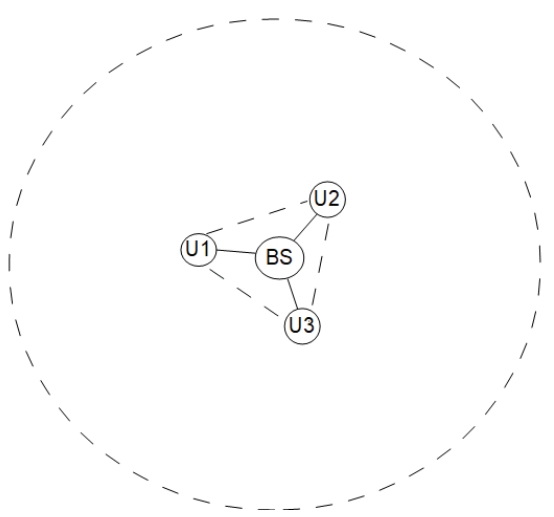

Fig. 3. Case 1

The second case is depicted in figure 4 . In this case all users are placed far from $\mathrm{BS}$ and at equal distances far from each other. 


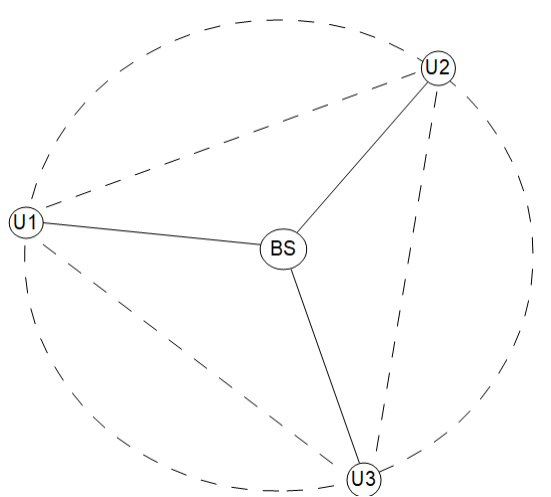

Fig. 4. Case 2

The third case considers two users that are placed close to each other but third user is far from them. All users are far from BS (Figure 5).

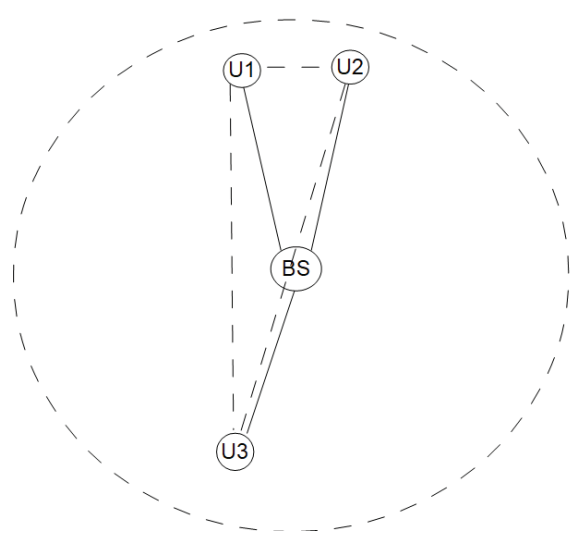

Fig. 5. Case 3

The location of users in fourth case is far from BS but they are close to each other. It is shown in Figure 6.

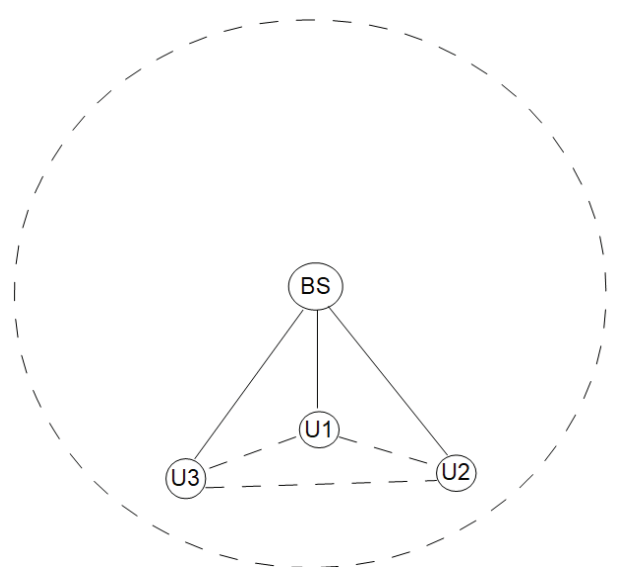

Fig. 6. Case 4

In each case 100 random matrix I realizations were generated. For example, first matrix I realization can be U1 transmits to U2, U2 transmits to U3, and U3 transmits to Internet. Second realization can be $\mathrm{U} 2$ transmits to U1, U1 transmits to U3. Base on these realizations it can be estimated used D2D routes percentage. This percentage for each case of user location is shown in Figure 7.

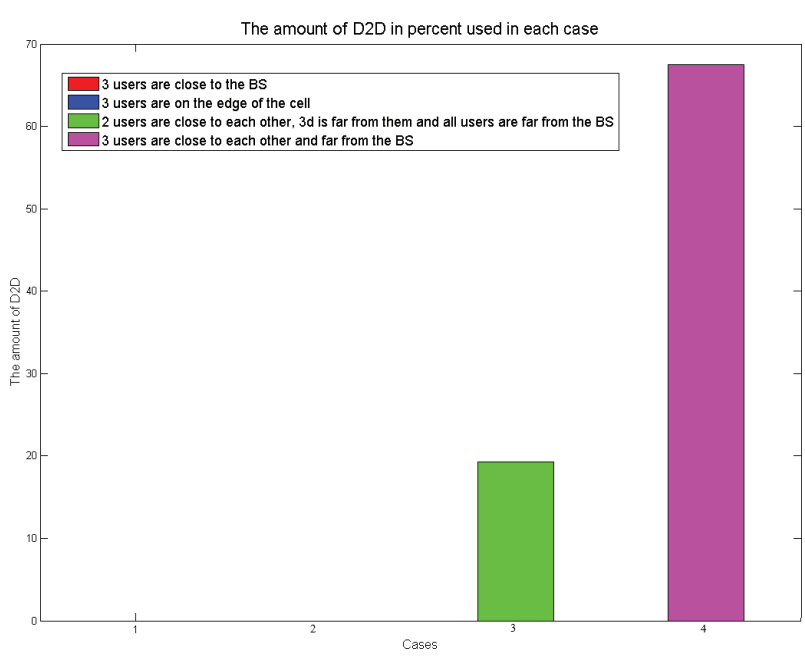

Fig. 7. D2D utilization versus case

According to this dependent, first and second cases use only routes transmitted data through BS (classical approach). Third case use about 19 percent of the maximum number of $\mathrm{D} 2 \mathrm{D}$ routes that can be used. In fourth case roughly 67 percent are used. So, the most beneficial case for D2D communication usage is fourth case. Of course, numbers in each case can be varied. It depends on users' location and matrix I realizations.

The next question is how does the data delivery time change when using D2D communications compared to using only the classical approach? To answer it, it will be considered two models: with the using only classical approach and the classical approach and D2D communications. Using these models, the D2D utilization gain as the ratio of the delivery time of the classical approach and D2D for the delivery time of only the classical approach is calculated. It's gain was calculated in each combination and then it was averaged.The average D2D utilization gain for the same cases of placed users and matrix $\mathbf{I}$ realizations are shown in Figure 8 for the transmitted data size of which is medium, i.e. less or equal to $1 \mathrm{Mb}$ and in Figure 9 for the transmitted data size of which is large, i.e. between 8 and $12 \mathrm{Mb}$.

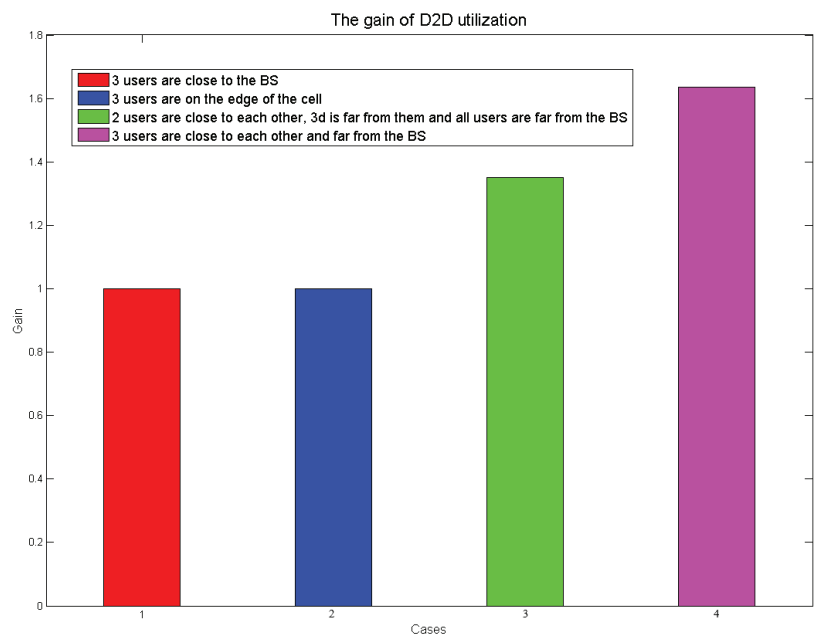

Fig. 8. D2D utilization gain when the transmitted data size is medium 


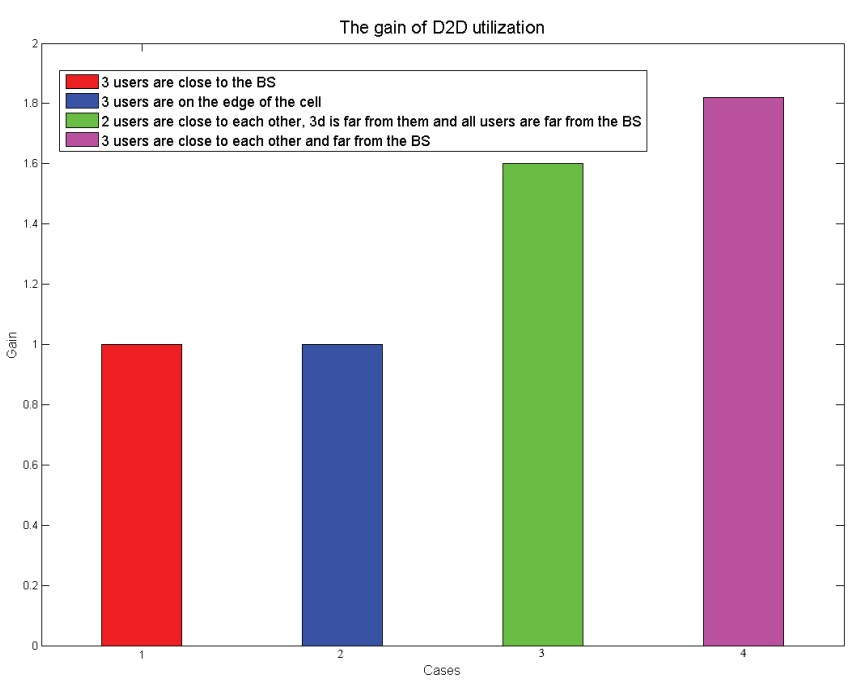

Fig. 9. D2D utilization gain when the transmitted data size is large

So, if gain equal to 1 , there is no benefit to use D2D as it can be seen in case 1 and case 2. Gain shows how much on average the using one combination of the classical approach and D2D in comparison with the classical approach only gives a benefit.

Since $\widetilde{I}_{g n^{R} n^{T}}$ is constant, the data delivery time depends on their size, which also affects the D2D utilization gain. The figures' 8 and 9 analysis shows that D2D utilization gain increases with an increase in the transmitted data size.

\section{CONCLUSION}

According to the results, there is no need to use the D2D connection when the distance between all users and the BS, as well as the distance between all users are approximately the same, which corresponds to cases 1 and 2. D2D gives a benefit when users are far from the $\mathrm{BS}$ and close to each other. In the 3rd case, when 2 users were close to each other, the $\mathrm{D} 2 \mathrm{D}$ utilization gain is 1.35 when the transmitted data size is medium, and 1.6 when the transmitted data size is large, whereas in the 4th case, when 3 users are located close to each other, the gain is 1.63 if the transmitted data size is medium, and 1.82 if the transmitted data size is large.

Moreover, the idea of using the collective dynamic routing method for allocating resource blocks was presented and implemented with using Bounded Recurrent Algorithm in Routing Stage. It allowed increasing data rates by reducing the intra-system interference flow in real systems due to its low computational complexity.

\section{References}

[1] E.A. Spirina Optimizacija raspredelenija informacii v fiksirovannyh setjah shirokopolosnogo radiodostupa s uchetom vnutrisistemnyh pomeh. Zhurnal radiojelektroniki [jelektronnyj zhurnal]. 2015. No $9 . \quad$ at http://jre.cplire.ru/jre/sep15/5/text.pdf. (in Russian)

[2] E.A. Spirina, S.V. Kozlov. A routing method providing throughput increase in IP networks with intrasystem interference. Zhurnal radiojelektroniki [jelektronnyj zhurnal]. 2015. No 12. Ava

http://jre.cplire.ru/jre/dec15/3/text.pdf. (in Russian)

[3] Ju.S. Vintenkova, S.V. Kozlov, E. A. Spirina. Analiz jeffektivnosti metoda sovmestnoj dinamicheskoj marshrutizacii $\mathrm{v}$ setjah shirokopolosnogo radiodostupa s trafikom protokolov TCP, HTTP, FTP. Zhurnal radiojelektroniki [jelektronnyj zhurnal]. 2016. No 1 . http://jre.cplire.ru/jre/jan16/5/text.pdf. (In Russian)

[4] Recommendation ITU-R P.1411-10. Propagation data and prediction methods for the planning of short-range outdoor radiocommunication systems and radio local area networks in the frequency range $300 \mathrm{MHz}$ to $100 \mathrm{GHz}$. Electronic Publication, Geneva, 2019, p. 52.

[5] Ministry of digital development, communications and mass communications of the russian federation, order № 572 on approval of the rules for application of basic stations and relays of networks of mobile radiotelephone communications. Part VI. Rules for application of basic stations and relays of networks of mobile radiotelephone communications of LTE standard and its modification LTE-advanced. Link: https://rulaws.ru/acts/PrikazMinkomsvyazi-Rossii-ot-29.10.2018-N-572/. (in Russian)

[6] S.V. Kozlov, E.A. Spirina, "Application efficiency analysis of the optimal measurement algorithm for method OFDM communication networks integrated optimization", 2019 Systems of Signal Synchronization, Generating and Processing in Telecommunications, SYNCHROINFO 2019. DOI: 10.1109/SYNCHROINFO.2019.8813911

[7] S.V. Kozlov, E.A. Spirina, L.I. Fazylova, "Svidetel'stvo o gosudarstvennoj registrac i programmy dlja JeVM №2016663494. Programma OFDM Receiver" - Zajavka №2016661064; Registered: 18.12.2016. (In Russian)

[8] Y.S. Vintenkova, S.V. Kozlov, E.A. Spirina. Bran collective dynamic routing optimal routes evaluation algorithm. 2018 Systems of Signals Generating and Processing in the Field of on Board Communications, 2018-January, 2018, pp. 1-3. DOI: 10.1109/SOSG.2018.8350578 